\title{
A seroepidemiological investigation of Lyme disease in Qiongzhong County, Hainan Province in 2019-2020
}

\author{
Shu Wen ${ }^{1 \#}$, Qianyan Xu ${ }^{1 \#}$, Dingfa Liu ${ }^{1}$, Zhangli Lin ${ }^{1}$, Zhishen Lin ${ }^{1}$, Shaojin Chen ${ }^{2}$, Mingwei Chen ${ }^{3}$ \\ ${ }^{1}$ Department of Laboratory Medicine, Shanghai Jiao Tong University Affiliated Tongren Hospital Hainan Branch/Qiongzhong Li and Miao \\ Autonomous County People's Hospital, Qiongzhong, China; '2Department of Laboratory Medicine, Sanya People’s Hospital, Sanya, China; \\ ${ }^{3}$ Department of Internal Medicine, Shanghai Jiao Tong University Affiliated Tongren Hospital Hainan Branch/Qiongzhong Li and Miao \\ Autonomous County People's Hospital, Qiongzhong, China \\ Contributions: (I) Conception and design: S Wen; (II) Administrative support: None; (III) Provision of study materials or patients: Q Xu; (IV) \\ Collection and assembly of data: D Liu, Z Lin; (V) Data analysis and interpretation: Z Lin; (VI) Manuscript writing: All authors; (VII) Final approval \\ of manuscript: All authors. \\ \#These authors contributed equally to this work. \\ Correspondence to: Shaojin Chen. Department of Laboratory Medicine, Sanya People's Hospital, Sanya, China. Email: 290322561@qq.com; Mingwei \\ Chen. Department of Internal Medicine, Shanghai Jiao Tong University Affiliated Tongren Hospital Hainan Branch/Qiongzhong Li and Miao \\ Autonomous County People’s Hospital, Qiongzhong, China. Email: 971909729@qq.com.
}

Background: The incidence and prevalence of Lyme disease are expanding and rising, respectively, which constitutes a worldwide public health problem. The present study aimed to investigate the prevalence of Lyme disease (LD) in Qiongzhong County, Hainan, and to determine the LD infection level in the local population, so as to provide evidence for further local LD surveillance as well as effective prevention and treatment measures.

Methods: A total of 252 serum samples were collected from cases with suspected LD in Qiongzhong County, including 159 and 93 cases with joint and neurological symptoms, respectively. The serum samples were preliminarily screened for LD antibodies by indirect immunofluorescent assay (IFA). Western Blot (WB) was used to confirm the diagnosis of IFA positive serum samples.

Results: Of the 252 serum samples, 39 tested positive by indirect immunofluorescence, translating to a positive rate of $15.48 \%$. Of these 39 positive serum samples, western blot testing confirmed 28 to be positive for LD antibodies, representing a positive rate of $11.11 \%$. LD spirochete infection was found in individuals of different ages, with the positive rate being highest among those aged $\leq 20$ and $\geq 81$ years old $(22.22 \%$ in both groups). The positive rate of LD antibody detection in men was $16.89 \%$, which was higher than the rate of $13.46 \%$ seen in women; however, the difference was not statistically significant. The positive rate of LD antibody detection using the 2-step testing method was $10.06 \%$ in patients with joint symptoms, which was slightly lower than the positive rate among individuals with neurological symptoms.

Conclusions: Our results confirm the presence of LD among the population of Qiongzhong County, Hainan. LD surveillance should be carried out to actively prevent and treat LD in the locality. Local doctors should also consider the possibility of LD when diagnosing and treating patients with arthritis and neurological diseases.

Keywords: Lyme disease (LD); seroepidemiological investigation; joint; neurological

Submitted Mar 01, 2021. Accepted for publication Apr 23, 2021.

doi: 10.21037/apm-21-693

View this article at: http://dx.doi.org/10.21037/apm-21-693 


\section{Introduction}

Lyme disease (LD) is a vector-transmitted disease resulting from Borrelia Burgdorferi (BB) bacterial infection (1). In the early stage of infection, LD manifests as skin migratory erythema, flu-like symptoms, arthralgia, muscle pain, chest pain, and other symptoms, and can later progress to facial neuritis, meningitis, facial nerve palsy, limb numbness, and other symptoms of nervous system damage (2). LD is known as the second form of AIDS in the United States. LM has been reported in more than 70 countries in Asia, Europe, America, Oceania, and Africa, and its incidence area and prevalence are expanding and rising, respectively. Thus, LM constitutes a worldwide public health issue $(3,4)$.

$\mathrm{BB}$ has been detected in patients, animals, and/or ticks in 30 autonomous regions and municipalities of China, confirming that the country is a natural foci of LD (5). As an endemic infectious disease, LD has also shown a trend of regional clustering in China, mainly in the Northwest, Inner Mongolia, and Xinjiang $(6,7)$. There are some reports in the literature of LD cases in southern regions of China; however, these reports are few compared to those in northern areas. A seroepidemiological survey conducted by Li et al. confirmed the presence of LD in the northeastern part of Hainan, an island province located in the South China Sea; however, no etiological study has confirmed that this area is a natural foci of LD (8). Through gene sequencing analysis, our research team found that Boophilus ticks in the Qiongzhong area carry multiple genotypes of $\mathrm{BB}$ and that the carrying rate is high, meaning there is a risk of LD transmission (9). Qiongzhong County is located in central Hainan, and its environment differs geographically and climatically from those of mainland provinces. Therefore, the present study aimed to investigate the prevalence of LD in Qiongzhong County and determine the infection level of LD in the local population, so as to provide a basis for further local LD surveillance as well as effective prevention and treatment measures. We present the following article in accordance with the MDAR reporting checklist (available at http:// dx.doi.org/10.21037/apm-21-693).

\section{Methods}

\section{Collection of serum samples}

From 2019 to 2020, 252 serum samples were collected from suspected LD cases in Qiongzhong County, including 159 cases with joint symptoms and 93 cases with neurological symptoms. Cases with rheumatoid arthritis or other diseases were excluded. The 148 males and 104 females included in the study ranged in age from 5 to 92 years old.

All procedures performed in this study involving human participants were in accordance with the Declaration of Helsinki (as revised in 2013). The study was approved by Qiongzhong Li and Miao Autonomous County People's Hospital (No.:20190541). Individual consent for this retrospective analysis was waived.

\section{Main reagents}

Fluorescein isothiocyanate-labeled goat anti-human immunoglobulin $M(\operatorname{IgM})$ and immunoglobulin $\mathrm{G}(\mathrm{IgG})$ antibodies, and horseradish peroxidase (HRP)-labeled goat anti-human IgM were obtained from Sigma (Milwaukee, Wisconsin, USA). HRP-labeled SpA was purchased from Borsi Biotechnology Co., Ltd. (Shanghai, China). LD spirochete standard strain PD91 immune rabbit serum, LD antigen sheets, and Western blotting (WB) film strips were supplied by the Lyme Ward of the Infectious Disease Control and Prevention Institute of the Chinese Center for Disease Control and Prevention.

\section{LD antibody test}

According to the recommendations of the Centers for Disease Control and Prevention (CDC) in the United States, a 2-step method was employed to detect antibodies to LD in patients' serum: step 1 was a primary screening test by indirect immunofluorescence assay (IFA), and step 2 was a confirmatory test by WB.

\section{IFA}

The serum was diluted with $3 \%$ egg yolk to a ratio of 1:16. A positive result was taken as more than $50 \%$ of the spirochetes being stained with bright fluorescence under the $40 \times 10$ field of the fluorescence microscope, with an antibody titer of $\operatorname{IgM} \geq 1: 64$ and $\operatorname{IgG} \geq 1: 128$. Positive and negative controls were made at the same time.

\section{Western blot (WB)}

Specimens that tested positive in the initial screening by IFA were subjected to confirmatory testing by WB. Serum was diluted with phosphate-buffered saline with Tween at a ratio of 1:25. The antigen strips were soaked in serum, shaken for 4 hours, and washed 3 to 5 times for 5 to 10 minutes each time. Then, the antigen strips were added to the secondary IgG or IgM antibody overnight. After elution 
( 5 times, for 5 to 10 minutes each time), color reagent was added for 30 minutes for color development, after which, the reaction was terminated with pure water. The results of WB were determined according to the Chinese WB diagnostic criteria for LD (10).

\section{Statistical analysis}

All statistical calculations were performed with SPSS 20.0 software. Data were analyzed using the chi-square $\left(\chi^{2}\right)$ test, and the results are expressed as percentages. $\mathrm{P}<0.05$ was considered as statistically significant.

\section{Results}

\section{LD serological antibody test results}

A total of 252 serum samples were tested by the IFA method. Of the 39 samples found to be positive for LD antibodies, 8 were $\operatorname{IgG}$ positive and 31 were $\operatorname{IgM}$ positive. Therefore, the positive rate of LD antibody detection by IFA was $15.48 \%$ (Table 1). As shown in Figure 1, compared with the negative control, the spirochete morphology was clearly visible when positive serum was diluted at a ratio of 1:64. For serum specimens that had a positive IFA result,

Table 1 IFA test results of 252 serum samples

\begin{tabular}{lccc}
\hline Antibody type & Samples & Positive & Positive rate (\%) \\
\hline IgG & 252 & 8 & 3.17 \\
IgM & 252 & 31 & 12.30 \\
Total & 252 & 39 & 15.48 \\
\hline
\end{tabular}

IFA, indirect immunofluorescence assay.
WB was performed for confirmatory testing. As shown in the results in Table 2, 28 copies were positive for LD antibodies, translating to a positive rate of $11.11 \%$. Among the 28 positive specimens were 12 cases with neurological symptoms and 16 cases with joint symptoms.

\section{Infection status by sex}

Among the 148 males included in the study, the positive rate of LD antibody detection using the IFA method was $16.89 \%(25 / 148)$, which was higher than the rate of $13.46 \%$ seen among females (14/104), although the difference was not statistically significant $\left(\chi^{2}=0.404, \mathrm{P}=0.52\right)$. The overall positive rate of LD antibody detection using the 2-step (IFA and WB) method was $10.81 \%$ for males and $11.54 \%$ for females (Table 3), and the difference was not statistically significant $\left(\chi^{2}=0.026, \mathrm{P}=0.87\right)$.

\section{Infection status by age}

Of the 252 specimens tested, positive results were observed in all age groups, with the ages of patients with LD antibody-positive serum specimens ranging from 5 to 92 years old. As shown in Table 4, after preliminary testing by IFA, the positive rates in the $\leq 20$ years, 21 to 40 years, 41 to 60 years, 61 to 80 years, and $\geq 81$ years age groups were $22.22 \%(14 / 63), 10.87 \%$ (5/46), $12.16 \%$ (9/74), $11.76 \%(6 / 51)$, and $22.22 \%$ (4/18), respectively. There was no significant difference in the LD antibody detection rate among the five age groups $\left(\chi^{2}=3.435, \mathrm{P}=0.49\right)$. The overall positive rates using the 2-step (IFA and $\mathrm{WB}$ ) method were $11.11 \%$ (7/63), $10.87 \%$ (5/46), $10.81 \%$ (8/74), $11.76 \%$ $(6 / 51)$, and $11.11 \%(2 / 18)$ in the $\leq 20$ years, 21 to 40 years, 41 to 60 years, 61 to 80 years, and $\geq 81$ years age groups,
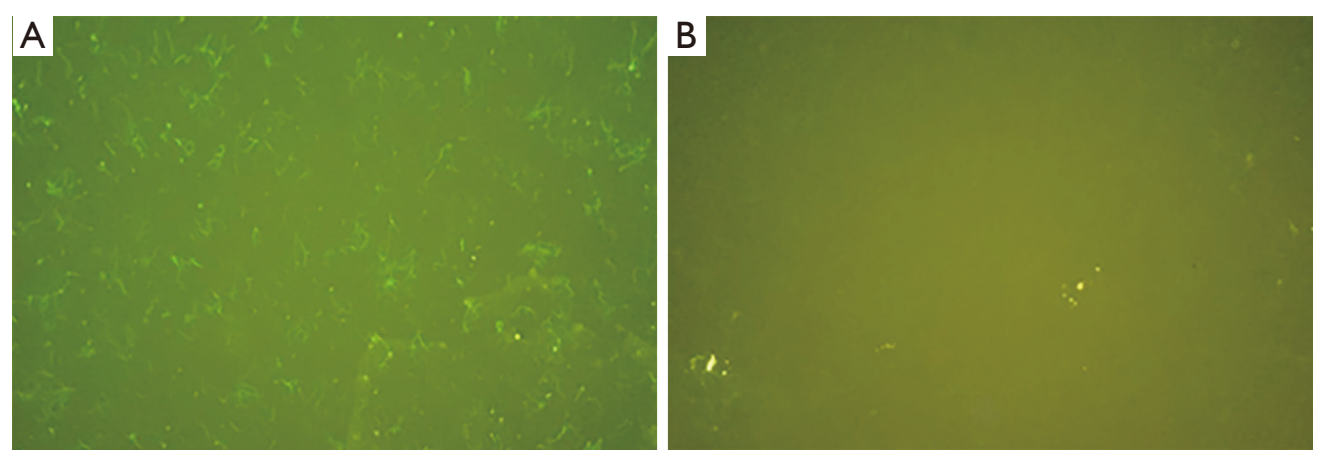

Figure 1 Fluorescence photos of serum IFA detection of LD antibodies (immunofluorescent staining, 400×). (A) A representative positive result and (B) the negative control. IFA, indirect immunofluorescence assay; LD, Lyme disease. 
Table 2 WB test results of 39 IFA-positive specimens

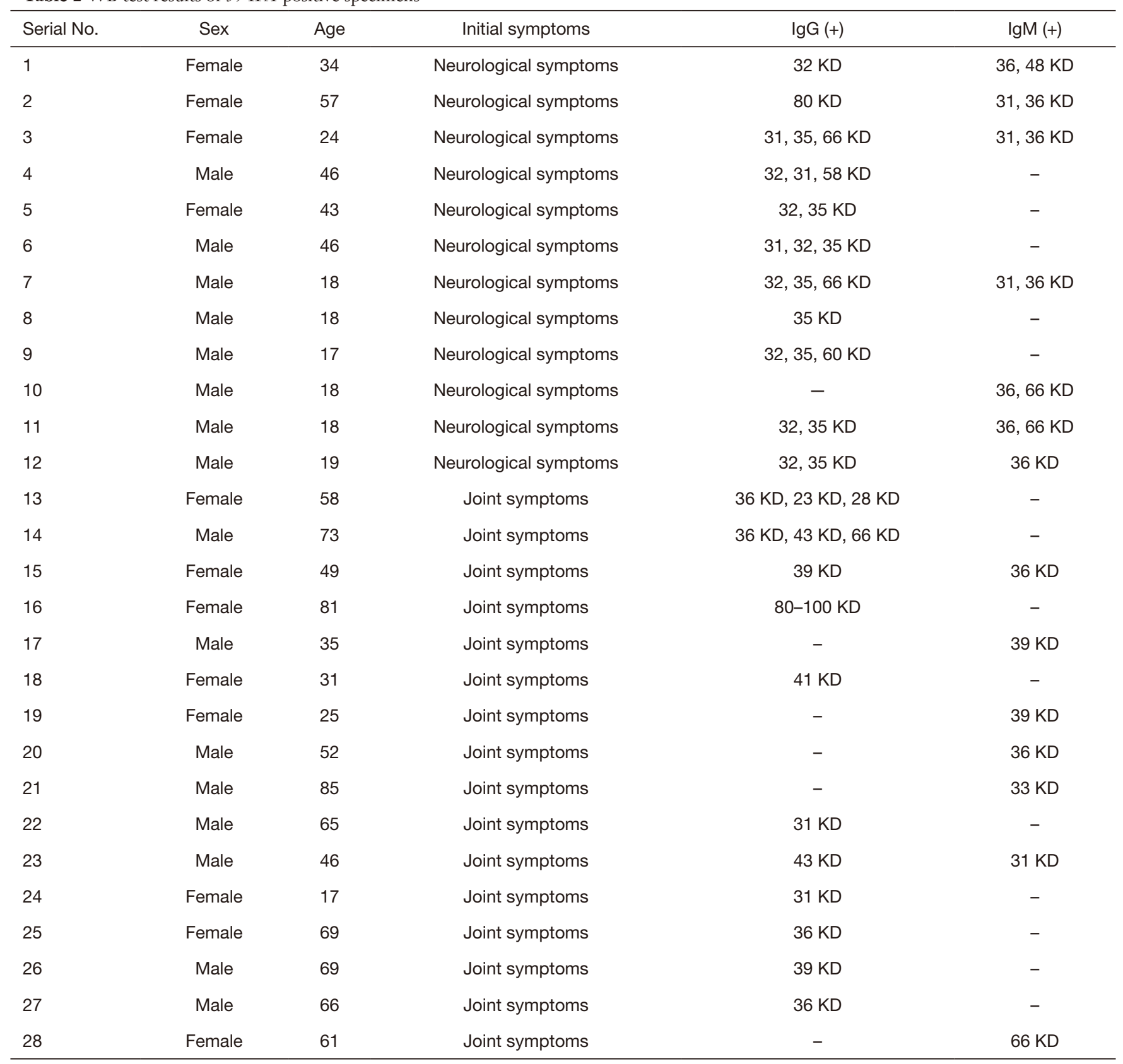

WB, Western blotting; IFA, indirect immunofluorescence assay.

Table 3 Results of LD serum antibody detection by sex

\begin{tabular}{|c|c|c|c|c|c|}
\hline Sex & No. of samples & \multicolumn{2}{|c|}{ IFA method } & \multicolumn{2}{|c|}{ WB method } \\
\hline Male & 148 & 25 & 16.89 & 16 & 10.81 \\
\hline Female & 104 & 14 & 13.46 & 12 & 11.54 \\
\hline
\end{tabular}

LD, Lyme disease; IFA, indirect immunofluorescence assay; WB, Western blotting. 
Table 4 Results of LD serum antibody detection by age

\begin{tabular}{|c|c|c|c|c|c|}
\hline Age group & No. of samples & \multicolumn{2}{|c|}{ IFA method } & \multicolumn{2}{|c|}{ WB method } \\
\hline$\leq 20$ years & 63 & 14 & 22.22 & 7 & 11.11 \\
\hline $21-40$ years & 46 & 5 & 10.87 & 5 & 10.87 \\
\hline $41-60$ years & 74 & 9 & 12.16 & 8 & 10.81 \\
\hline$\geq 81$ years & 18 & 4 & 22.22 & 2 & 11.11 \\
\hline
\end{tabular}

LD, Lyme disease; IFA, indirect immunofluorescence assay; WB, Western blotting.

Table 5 Results of LD serum antibody detection in cases with different symptoms

\begin{tabular}{lcccccc}
\hline \multirow{2}{*}{ Symptom type } & \multirow{2}{*}{ No. of samples } & \multicolumn{2}{c}{ IFA method } & \multicolumn{2}{c}{ WB method } \\
\cline { 3 - 5 } \cline { 5 - 6 } & & No. of positives & Positive rate (\%) & & No. of positives & Positive rate (\%) \\
\hline Joint & 159 & 20 & 12.58 & 16 & 10.06 \\
Neurological & 93 & 19 & 20.43 & 12 & 12.90 \\
\hline
\end{tabular}

LD, Lyme disease; IFA, indirect immunofluorescence assay; WB, Western blotting.

respectively. There were no statistically significant differences in the LD antibody detection rates among the different age groups.

\section{Infection status of cases with different symptoms}

The rates of $\mathrm{LD}$ antibody detection were also examined in different symptomatic groups (Table 5). The positive rate of LD antibody detection using the IFA method was $20.43 \%$ (19/93) in cases with neurological symptoms, which was higher than the positive rate of $12.58 \%(20 / 159)$ observed among cases with joint symptoms, although the difference was not statistically significant $\left(\chi^{2}=1.99, \mathrm{P}=0.16\right)$. The overall positive rate of $\mathrm{LD}$ antibody detection using the 2 -step (IFA and WB) method was $10.06 \%$ in patients with joint symptoms, which was slightly lower than the positive rate seen among people with neurological symptoms $(12.90 \%)$; however, the difference was not statistically significant $\left(\chi^{2}=0.38, P=0.54\right)$.

\section{Discussion}

LD is a typical natural foci disease, which can circulate in nature without any reliance on humans. Any area with a suitable ecological environment and biological community may form a natural foci of $\mathrm{LD}$. The prevalence of $\mathrm{LD}$ is restricted by the natural geographic landscape and human factors. The more opportunities for host animals and vector ticks to come into contact humans, the more likely infections are to occur (11). However, in China, LD is not a legally reported infectious disease, so the disease is not well known, and the infection status among the populations of different areas is unclear.

Scholars from various countries believe that the main biological vectors of LD spirochetes differ between animals and humans. Zhang et al. discovered that Boophilus ticks in the Qiongzhong area carried multiple genotypes of $\mathrm{BB}$ and that the carrying rate was high, thus confirming the risk of LD transmission (9). Hainan is an island province located off the southernmost tip of mainland China. The natural geographical conditions in Hainan are extremely different from other areas of China with a high incidence of LD. In their study, $\mathrm{Li}$ et al. (12) conducted LD antibody screening of serum from patients with joint and neurological symptoms in Sanya City, Hainan, and confirmed the presence of LD in the province. Currently, the treatment of Lyme disease mainly includes antibiotics, non-steroidal antiinflammatory drugs, and glucocorticoids. Antibiotics, such as tetracycline, amoxicillin, penicillin, and doxycycline, are effective against various lesions of LD. Nonsteroidal antiinflammatory drugs (NSAIDs) are used to treat arthritis in Lyme disease, such as Fenbid. Glucocorticoids are indicated 
for patients with Lyme disease meningitis or carditis, such as secreisone.

The results of the present study showed that among 252 serum samples, the positive rate of antibody detection using the IFA method was $15.48 \%$, while the positive rate using the WB method was $11.11 \%$. The difference in positive rate between the two methods may be attributed to the crossreaction between IFA antibody and Leptospira, Treponema pallidum and other Treponema antibodies, resulting in false positive results. In 2010, the Biesiada (13) study showed that to improve the specificity and diagnostic accuracy in the detection of LD, a 2-step method is required, comprising initial screening by enzyme-linked immunosorbent assay or IFA, followed by WB for diagnostic confirmation of preliminarily positive results.

In the present study, the results of sex and age-based analyses showed that LD spirochetes were detected in both males and females, and in all age groups. The positive rate was the highest among cases aged $\leq 20$ and $\geq 81$ years old (both $22.22 \%$ ), and the antibody detection rate was higher among men than among women (16.89\% vs. $13.46 \%)$, although the difference was not statistically significant. The LD serological survey conducted in the Miyun District of Beijing by Yang et al. showed that the total positive rate of antibodies in women was higher than that in men, and that the 50 years old age group had the highest overall positive antibody detection rate (14). The findings of $\mathrm{Li}$ et al. were consistent with the results of the present study; they found that there was no statistically significant difference in the LD positive antibody rate among patients of different sexes and ages in 3 hospitals in the northeastern part of Hainan province (8). In this study, LD antibodies were detected in serum of cases of suspected LD in Qiongzhong County, Hainan, which indicates that LD is present in the local population. The positive rate of total antibodies to LD in cases with neurological symptoms using the IFA method was $20.43 \%$, which was higher than the positive rate of $12.58 \%$ observed in cases with joint symptoms. The overall positive rate of antibodies to LD in cases with joint symptoms using the 2-step (IFA and WB) method was $10.06 \%$, which was slightly lower than the positive rate in cases with neurological symptoms (12.90\%).

LD is a syndrome associated with multiple system damage. In addition to chronic skin migrating erythema, heart damage, and joint symptoms, patients can also experience complex neurological symptoms (15). Nervous system damage is among the most serious clinical manifestations of LD. BB is highly neurotropic; not only can it cause neurological symptoms, but it can also incubate in the central nervous system over the long term, showing different neural spirochetes at different stages. The disease seriously affects patients' quality of life. This study has evidenced the existence of LD in patients with joint and neurological symptoms in Qiongzhong County, Hainan, which should prompt local clinicians to consider the possibility of LD in patients presenting with such symptoms.

This study found evidence that LD is present in Qiongzhong County, Hainan. However, the number of samples in this study was limited, meaning our results cannot accurately reflect the prevalence of LD in the local population. Nevertheless, our study provides a theoretical basis for the prevention and control of LD in Qiongzhong County. Local medical staff should pay attention to the differential diagnosis of LD in patients with neurological and joint symptoms, so as to ensure early detection, diagnosis, and treatment to control the occurrence of LDrelated complications. At the same time, attention should be paid to the dissemination of LD knowledge among forest workers, forest guards, farmers, and young people, to increase the awareness of the local population. Through such measures, we can reduce tick exposure and the chances of sustaining a tick bite, and prevent and control the occurrence of LD.

\section{Acknowledgments}

Funding: This research was supported by the Hainan Province Health and Family Planning Industry Research Project (No. 19A200039).

\section{Footnote}

Reporting Checklist: The authors have completed the MDAR reporting checklist. Available at http://dx.doi.org/10.21037/ apm-21-693

Data Sharing Statement: Available at http://dx.doi. org/10.21037/apm-21-693

Conflicts of Interest: All authors have completed the ICMJE uniform disclosure form (available at http://dx.doi. org/10.21037/apm-21-693). The authors have no conflicts of interest to declare.

Ethical Statement: The authors are accountable for all 
aspects of the work in ensuring that questions related to the accuracy or integrity of any part of the work are appropriately investigated and resolved. All procedures performed in this study involving human participants were in accordance with the Declaration of Helsinki (as revised in 2013). The study was approved by Qiongzhong Li and Miao Autonomous County People's Hospital (No.:20190541). Individual consent for this retrospective analysis was waived.

Open Access Statement: This is an Open Access article distributed in accordance with the Creative Commons Attribution-NonCommercial-NoDerivs 4.0 International License (CC BY-NC-ND 4.0), which permits the noncommercial replication and distribution of the article with the strict proviso that no changes or edits are made and the original work is properly cited (including links to both the formal publication through the relevant DOI and the license). See: https://creativecommons.org/licenses/by-nc-nd/4.0/.

\section{References}

1. Lin S, Shrestha S, Prusinski MA, et al. The effects of multiyear and seasonal weather factors on incidence of Lyme disease and its vector in New York State. Sci Total Environ 2019;665:1182-8.

2. Petrulionienė $\mathrm{A}$, Radzišauskienè $\mathrm{D}$, Ambrozaitis $\mathrm{A}$, et al. Epidemiology of Lyme Disease in a Highly Endemic European Zone. Medicina (Kaunas) 2020;56:115.

3. Moon S, Hong Y, Hwang KJ, et al. Epidemiological features and clinical manifestations of Lyme borreliosis in Korea during the period 2005-2012. Jpn J Infect Dis 2015;68:1-4.

4. Piesman J. Strategies for reducing the risk of Lyme borreliosis in North America. Int J Med Microbiol 2006;296 Suppl 40:17-22.

5. Zhang AQ, Shen ZY, Liu ZJ, et al. Research progress on epidemiology of Lyme disease. Chin Hyg Insert \& Equip 2020;26:79-83.

Cite this article as: Wen S, Xu Q, Liu D, Lin Z, Lin Z, Chen S, Chen M. A seroepidemiological investigation of Lyme disease in Qiongzhong County, Hainan Province in 2019-2020. Ann Palliat Med 2021;10(4):4721-4727. doi: 10.21037/apm-21-693
6. Wang B, Ma CP, Zhu WF, et al. Investigation of seroepidemiology of Lyme disease and related factors in some regions of Gansu province. Medical Journal of National Defending Forces in Southwest China 2020;30:971-2.

7. Wu JY, Gong TM, Wang L, et al. Investigation on serum epidemiology of tick-born Lyme disease in different population in Kashi city of Xinjiang. J Trop Med 2017;17:1663-5.

8. Li S, Zhang L, Zhu X, et al. Test results for detection of antibody against Lyme disease in certain patients in the northeast of Hainan province. Pract Prev Med 2020;27:1068-71.

9. Zhang L, Wen S, Zhu X, et al. Detection and genotyping of Borrelia burgdorferi in parasitic ticks in Qiongzhong area of Hainan province, China. Chin J Vector Biol \& Control 2020;31:426-9.

10. Liu ZY, Hao Q, Hou XX, et al. A study of the technique of western blot for diagnosis of lyme disease caused by Borrelia afzelii in China. Biomed Environ Sci 2013;26:190-200.

11. Best SJ, Tschaepe MI, Wilson KM. Investigation of the performance of serological assays used for Lyme disease testing in Australia. PLoS One 2019;14:e0214402.

12. Li S, Chen T, Zhu X, et al. Analysis of serum screening for Lyme disease in patients with neurological symptoms in Sanya. Lab Med Clin 2018;15:1465-7.

13. Ross Russell AL, Dryden MS, Pinto AA, et al. Lyme disease: diagnosis and management. Pract Neurol 2018;18:455-64.

14. Yang YS, Lv YN, Chen YL. Serological investigation in arthritis patients with Lyme disease in Miyun district, Beijing. Chin J Vector Biol \& Control 2017;28:490-1.

15. Nelder MP, Wijayasri S, Russell CB, et al. The continued rise of Lyme disease in Ontario, Canada: 2017. Can Commun Dis Rep 2018;44:231-6.

(English Language Editor: J. Reynolds) 